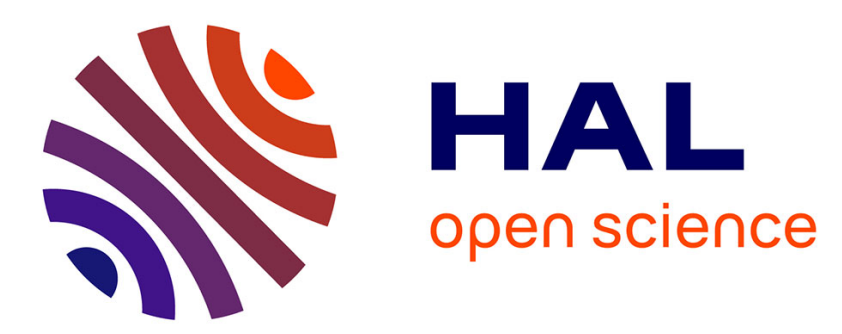

\title{
Influence of the Punch Speed on the Die Wall/Powder Kinematic Friction During Tableting
}

\author{
Léo Desbois, Pierre Tchoreloff, Vincent Mazel
}

\section{To cite this version:}

Léo Desbois, Pierre Tchoreloff, Vincent Mazel. Influence of the Punch Speed on the Die Wall/Powder Kinematic Friction During Tableting. Journal of Pharmaceutical Sciences - Elsevier, 2019, 108, pp.3359 - 3365. 10.1016/j.xphs.2019.05.007 . hal-03487638

\section{HAL Id: hal-03487638 \\ https://hal.science/hal-03487638}

Submitted on 21 Dec 2021

HAL is a multi-disciplinary open access archive for the deposit and dissemination of scientific research documents, whether they are published or not. The documents may come from teaching and research institutions in France or abroad, or from public or private research centers.
L'archive ouverte pluridisciplinaire HAL, est destinée au dépôt et à la diffusion de documents scientifiques de niveau recherche, publiés ou non, émanant des établissements d'enseignement et de recherche français ou étrangers, des laboratoires publics ou privés.

\section{(ㄷ)(1) $\$$}

Distributed under a Creative Commons Attribution - NonCommercial| 4.0 International 
$4{ }^{1}$ Univ. Bordeaux, CNRS, I2M Bordeaux, 146 rue Léo Saignat 33000 Bordeaux

5 *corresponding author: Université de Bordeaux, CNRS, I2M Bordeaux, 146 rue Léo

6 Saignat, F-33000 Bordeaux, France ; tel:+33 557579260 ; E-mail address:

7 vincent.mazel@u-bordeaux.fr

8 Abstract

9 Influence of the compaction speed on the final tablet properties is an important 10 challenge during the scale-up of a solid dosage form. This strain rate sensitivity is 11 generally attributed to the time dependent deformation behavior of the powder. In this 12 work, we studied the influence of the speed on another important factor during compaction: friction between the tablet/powder and the die. magnesium stearate.

\section{friction during tableting}

\author{
Léo Desbois ${ }^{1}$, Pierre Tchoreloff ${ }^{1}$, Vincent Mazel ${ }^{1, *}$
}


25 Tableting is one the most important process for the manufacturing of drugs. Despite an apparent simplicity, the phenomena occurring during tableting are complex and neither fully described nor understood. Some classical manufacturing problems, like capping and sticking, even if known since more than a century, can still be industrial issues today. Among the challenges during the development of new tablets, scale-up is one of the most critical, because some problems may remain undetected during development. The main reason is often the difference between the tablet presses used in development and the presses used in production. The conditions of compression may be different between different kinds of presses, leading to different results in terms of tablet final properties. As a consequence, compaction simulators have been developed to fill the gap between development eccentric presses and large production rotary presses.

The main parameter that may change during the scale up from a development press to an industrial press is the compaction kinematic. In fact, on high capacity rotary presses with large turrets, the compaction speed can be very high with punch tip maximal speed much higher than on eccentric presses. It is well known that in a lot of 41 cases, the speed of the punches, i.e. the speed at which the pressure is applied, can have consequences of the final tablet quality. This phenomenon is generally called

43 Strain Rate Sensitivity (SRS), and has been widely studied in the literature ${ }^{1}$. Several 44 aspects can explain the SRS. First, the deformation behavior of the powder can 45 depend on the strain rate. Viscoelasticity corresponds to the changes of the elastic behavior and viscoplasticity corresponds to the changes of the plastic behavior with

47 the strain rate. Both aspects have been largely studied ${ }^{2-8}$. Another part that can 
49 increases, air can have difficulties to escape and may thus change the apparent 50 deformation behavior of the powder ${ }^{9,10}$.

51 The previous aspects refer to the deformation behavior of the powder. Nevertheless

52 more recently ${ }^{11}$ it was shown that the interaction between the die and the powder,

53 i.e. friction, can also be modified when the speed increases. In fact, the ejection force

54 was found dependent on the compaction speed. Sun proposed a mechanism to 55 explain this phenomenon. His hypothesis was that magnesium stearate was 56 migrating toward the die during compaction. At slow speed, it would have more time 57 to migrate toward the die. At high speed, lubrication would thus be less efficient. 58 Nevertheless, there was no definite proof in the cited study that the migration 59 magnesium stearate was indeed responsible of the friction variations. Studies 60 performed in other domains have also shown that the friction coefficient between two 61 surfaces can change when the sliding speed between the two surfaces changes ${ }^{12}$.

62 Concerning the friction coefficient, it is important to separate the static friction 63 coefficient from the kinematic coefficient of friction ${ }^{13}$. The static coefficient of friction 64 acts before the powder or the tablet starts moving. The kinematic coefficient of 65 friction acts when the powder is moving. These two coefficients can be different, especially if there is adhesion between the two surfaces. In all this work, we will study

67 the kinematic coefficient of friction.

68 The present work aims to study the influence of sliding speed on the kinematic 69 friction coefficient between the powder and the die-wall. A protocol of measurement 70 was defined in order to separate the compaction speed during the making of the 71 tablet and the punch speed during the measurement of the friction coefficient. 72 Moreover, external lubrication was also used, in order to have a level of magnesium 
73 stearate on the die wall independent of the punch speed. This made it possible to

74 isolate the dependence of the friction coefficient on the speed from the problem of magnesium stearate migration during compaction. At the end of the study, practical consequences of the variation of the friction coefficient are proposed.

\section{Material and method}

\subsection{Materials}

\subsubsection{Powders}

Experiments were performed on four classical pharmaceutical excipients: Lactose Monohydrate (GLac) (Excipress, ArmorPharma, Maen Roch, France), Spray-dried Mannitol (SDMan) (Pearlitol 200SD, Roquette, Lestreme, France), Anhydrous Calcium Phosphate (Dicafos A60, Budenheim, Budenheim, Germany) and Calcium sulfate (CaSul) (Compactrol, JRS Pharma, Rosenberg, Germany). External lubrication was performed using Magnesium Stearate (Partek Mg Lub, Merck, Darmstadt, Germany).

\subsubsection{Powder compaction}

All compaction experiments were performed on a compaction simulator Styl'One Evolution (Medelpharm, Beynost, France). This device is a single station instrumented tableting machine. It is equipped with force sensors (strain gauges) on the upper and lower punches and the displacements of the punches are monitored using incremental sensors. An instrumented die using strain gauge technology was used to monitor the die-wall pressure. All experiments were performed using round flat punches with a diameter of $11.28 \mathrm{~mm}$. To normalize the experiments and decrease variability, the filling height was monitored to obtain tablets with a final thickness of about $3 \mathrm{~mm}$. Exact compaction conditions are detailed below in this 


$$
\mu=\frac{F_{p}}{P_{r a d} \pi D h}
$$

115 where $\mu$ is the friction coefficient, $F_{p}$ is the force needed to move the tablet, $P_{\text {rad }}$ is the 116 radial stress applied to the tablet, $\mathrm{D}$ is tablet diameter and $\mathrm{h}$ is the tablet thickness. If 117 the peak force needed to start the movement is taken as $F_{p}, \mu$ will be the static 118 coefficient of friction. If the plateau force to move the tablet at a constant speed is 119 taken as $F_{p}, \mu$ will be the kinematic coefficient of friction. 
120 In his work, Sun used the peak ejection force for $F_{p}$. This value is related to the static 121 friction coefficient. The residual die wall pressure at the end of the decompression 122 was taken to estimate $P_{\mathrm{rad}}$ and the thickness at the end of the decompression to 123 estimate $\mathrm{h}$. This is an approximation, first because some relaxation may occur 124 between the end of the decompression and the ejection and second because due to 125 the ejection force, the tablet is slightly recompressed. This may affect the height and 126 the die wall pressure. The reason for this approximation was mainly due to the 127 technology used for die wall pressure measurement. When strain gauges are used, 128 the measurement of the die wall pressure is linked with the thickness of the tablet, 129 which can only be known when both punches are in contact with the tablet, which is 130 not the case during ejection. Die wall pressure can thus not be measured during 131 ejection.

132 To overcome this problem, we changed the way of measuring the friction. After the 133 first compression that made the tablet, the lower punch was moved down inside the 134 die. Then, the tablet was pushed down in the die by the upper punch moving at 135 constant speed. During this phase, a constant force can be achieved which 136 corresponds to the force needed to move the tablet at a constant speed. This force is 137 related with the kinematic coefficient of friction. The speed of the upper punch was 138 changed during this phase to study the influence of the speed on $F_{p}$. At some point 139 the tablet enters again in contact with the lower punch. At this moment, it is thus 140 possible to measure both the die wall pressure and the tablet thickness for a given $141 \mathrm{~F}_{\mathrm{p}}$. These values were then taken to calculate the kinematic friction coefficient 142 corresponding to the speed used to push the tablet using equation 1.

143 The protocol can be summarized as (Figure 1): 
(1) The tablet is made using a symmetrical compression under constant kinematic conditions and maximum applied pressure.

(2) The lower punch moves down and the upper punch is used to push the tablet down in the die. During this phase, $F_{p}$ is measured. The speed of the upper punch can be varied.

(3) When the tablet enters in contact with the lower punch, it is possible to measure the exact height of the tablet and the value of the die wall pressure.

151 During the phase where the tablet is pushed down in the die, it was possible to obtain 152 constant speeds from $0.25 \mathrm{~mm} / \mathrm{s}$ to $40 \mathrm{~mm} / \mathrm{s}$. Six different speeds were used in this 153 interval: $0.25,1,4,10,25$ and $40 \mathrm{~mm} / \mathrm{s}$. The exact value of the speed was measured 154 for each experiment. For each condition, three measurements were performed and 155 all the individual measurement points will be displayed on the following graphs.

\section{Results and discussion}

\subsection{Results}

\subsubsection{Evolution of the force needed to push the tablet $\left(F_{p}\right)$}

In the first part of the experiment, the force needed to push the tablet down in the die is measured. An example of signals obtained for the same product (SDMan) at three

161 different speeds can be seen in Figure 2. It can be seen that the force is fairly 162 constant. A small peak force at the beginning is only obtained for the lowest speed. 163 This means that in the condition used, especially thanks to the use of external 164 lubrication, adhesion between the powder and the die is very low. There is thus in our case no significant difference between the static and the kinematic coefficient of 166 friction. The force signal during the tablet movement is fairly constant, i.e. we can 167 obtain a stationary state. A mean value of the plateau was calculated and taken as 
$168 F_{p}$. Comparison for the three speeds gives a first idea of the evolution of the friction

169 with the speed. $F_{p}$ clearly increases when the sliding speed increases. It can be 170 noted that for the highest speed, the force seems to increase at the beginning and 171 decrease at the end. This is due to changes in the speed. In fact, at high speed, it 172 takes more time to reach the desire speed and the device need to decelerate earlier 173 to be able to stop at the right time. Constant speed is thus not obtained for the whole 174 movement of the tablet. In this case, $F_{p}$ is taken when the speed of the punch is 175 stabilized at the right speed and not on the whole plateau.

176 This experiment was performed for the four products, using two different compaction 177 pressures for the first compression (150 MPa and $250 \mathrm{MPa}$ ). The results of the 178 evolution of $F_{p}$ as a function of the different speeds can be seen in Figure 3.

179 For each product and for each compression point, the same trend is obtained. $F_{p}$ 180 increases when the punch speed increases. These results are coherent with those 181 presented by Sun ${ }^{11}$ who found an increase of the ejection force when the speed 182 increased. Nevertheless, for each speed, the tablet was obtained under the same 183 conditions and external lubrication guaranties a reproducible lubrication state. 184 Migration of Magnesium Stearate during compression is thus, in our case, not 185 responsible of the evolution obtained. We can also note that the evolution of $F_{p}$ with 186 the speed follows globally a logarithmic trend.

187 In more quantitative terms it is interesting to note that $F_{p}$ can be multiplied by factor 188 until 4, which means the effect of the sliding speed is far from being marginal. Of 189 course, the evolution of the force alone does not give a complete picture of the 190 phenomena. For example, it is not possible to compare the results between the 191 products and the different compaction pressures because the die wall pressure may 
192 change between the different situations. To go further, it is thus important to study the

193 evolution of the die wall pressure in order to be able to finally calculate the friction 194 coefficient.

\subsubsection{Estimation of the die wall pressure}

As explained above the die wall pressure is measured when the tablet reaches the lower punch. At the same moment the force on the upper punch is also measured. 198 For low speeds, this force is nearly equal to $F_{p}$, the force on the plateau. For high speeds, when the tablet touches the lower punch, the speed already began to decrease, and the force is lower than $F_{p}$. To be as coherent as possible, for the evolution of the wall pressure, the force considered for the upper punch was the one 202 measured when the tablet touches the lower punch.

203 The evolution of the die wall pressure as a function of the force on the upper punch 204 for the four products and two compression levels are presented in Figure 4. As 205 expected, different die wall pressures were obtained for the different products, 206 because this parameter is linked with the mechanical behavior of the powder. The 207 values obtained for a compaction pressure of $250 \mathrm{MPa}$ were higher than the values 208 obtained for $150 \mathrm{MPa}$ which was also expected.

210 For both compression pressure, the die wall pressure slightly increased with force 211 applied by the upper punch. This increase was generally between 1 and $2 \mathrm{MPa}$ on 212 the force range explored. This increase can be explained by the slight recompression 213 experienced by the tablet due to the force applied by the upper punch. It can be 214 approximately represented by a linear relationship. These linear relations were thus 
215 taken to calculate the value of the die wall pressure for each experiment in order to

216 be able to estimate the friction coefficient.

\subsubsection{Evolution of the friction coefficient}

218 Finally, it was possible for each experiment performed to estimate the friction 219 coefficient. All the results are presented in Figure 5.

220 The results indicate that for all the cases the friction coefficient increased when the

221 punch speed increased. These results confirm the findings of Sun ${ }^{11}$, but thanks to 222 our methodology, it is possible to affirm that, in our case, this evolution is not related 223 with the migration of magnesium stearate during the compression phase, as the 224 measurements for different speed were made on tablets obtained under the same 225 conditions. This evolution is an intrinsic property of the friction between a tablet and 226 a die lubricated with magnesium stearate. As for the $F_{p}$, the evolution of the friction 227 coefficient follows globally a logarithmic trend.

228 If we compare the products, it seems that SDMan, GLac and CaSul gave a similar 229 trend in terms of evolution. At low speed, they gave similar friction coefficients. At the 230 highest speeds the results are slightly different, especially SD Man seems to give 231 higher coefficients of friction. On the contrary, ACP has a different behavior with a 232 much smaller dependence to the speed. If we consider the tablets obtained under $233150 \mathrm{MPa}$, the increase of the friction coefficient for ACP was around $50 \%$ on the 234 speed range whereas the increase was around $200 \%$ for the other products.

235 Another interesting point is to compare the friction coefficients obtained for tablets compacted at $150 \mathrm{MPa}$ and $250 \mathrm{MPa}$. Changing the pressure used to make the

237 tablet has two main consequences. First it changes the structure of the tablet and, as 238 a consequence, it can change the structure of the interface between the tablet and 
239 the die. Second it changes also the value of the die pressure applied on the tablet.

240 Comparison of the coefficient of friction between the two situations for the four

241 products is presented in Figure 6. From this results it is difficult to obtain a general

242 trend. For SDMan and ACP, no influence of the first pressure is obtained. For CaSul

243 and GLac, the results seemed to indicate a slight decrease of the friction coefficient

244 when the pressure increases, especially at high speed. Nevertheless, for each

245 product, the influence of the first compaction is much lower than the influence of the 246 speed of the punch.

\section{$247 \quad$ 3.2. Discussion and consequences}

\subsubsection{Discussion}

249 The protocol defined above made it possible to confirm that the coefficient of friction

250 between the tablet and the die in a tableting machine is dependent on the sliding 251 speed between the tablet and the die, as observed by Sun. As the conditions to 252 make the tablet were kept constant for each sliding speed and external lubrication 253 was used, the increase in the friction coefficient can be considered as an intrinsic 254 property of the friction between a tablet and a die lubricated with magnesium 255 stearate.

256 Lubrication using magnesium stearate, is generally considered as a boundary 257 lubrication, i.e. magnesium stearate forms a thin film at the surface of the die that 258 interpose between the two sliding surfaces ${ }^{14}$. In this kind of situation, as the 259 lubricating film will reduce the surface interaction between the two sliding surface 260 (here the die and the product in the tablet), the friction is due almost entirely to the 261 shear strength of the lubricating film ${ }^{15}$. Several studies have shown that the shear 262 strength can be dependent on the shear rate, and as a consequence, the friction 
263 coefficient can be dependent on the sliding speed ${ }^{16}$. More precisely, Campen et al.

264 reviewed recently a number of published studies where the friction coefficient 265 increases with of the sliding speed according to a logarithmic trend ${ }^{12}$. Results 266 presented in Figure 5, are totally coherent with the cited literature as it shows that, in 267 the velocity range studied, the friction coefficient increases with the sliding speed in a 268 logarithmic way. This means that the shear strength of magnesium stearate is 269 dependent on the shear rate, as it was already demonstrated for thin layers of stearic 270 acid $^{17}$.

271 As noted before, CaSul, GLac and SDMan gave nearly the same result which is 272 understandable, as the friction is mainly govern by the properties of the magnesium 273 stearate film. Nevertheless, some contact between the two layers (excipient/metal) 274 may remain, which could explain that the values are not totally the same. The case of 275 ACP is different because it gave a different trend for the evolution of the friction 276 coefficient, with a higher value at low speed and a smaller sensitivity to the sliding 277 speed. To try to understand this different trend, the sliding surface of the tablets (i.e. 278 the tablet band) was observed using scanning electron microscope. Photographs of 279 the tablet band for the four products obtained under a pressure of $150 \mathrm{MPa}$ can be 280 observed in Figure 7. These images make it possible to compare, between the four 281 products, the effective contact surface between the tablet and the die.

282 GLac and SDMan gave relatively smooth tablet band and a more porous surface is 283 observed for CaSul. Nevertheless, for these three products, a large part of the band 284 is indeed in contact with the die. The situation is completely different for ACP. To 285 better visualize the surface, another photograph obtained with a higher magnification 286 is presented in Figure 8. The surface is far from being smooth and the structure of 287 the grains is still clearly visible on the photograph. It is easy to see the points that 
288 were in contact with the die, because they present a very smooth surface. Using 289 image analysis, it is possible to estimate that only around $10-15 \%$ of the apparent 290 surface was indeed in contact with the die. This means that, for example, the actual 291 local die-wall pressure is much higher than the value considered in this study (which 292 take into account the whole side surface). Even if it is difficult to propose a complete 293 mechanical explanation of the observed variation, this difference in contact surface 294 and pressure constitute a plausible explanation of the typical behavior of this product. 295 Finally, it must be noted that the grade of ACP used in this study (Dicafos A60) has very typical properties compared to other commercial available grades ${ }^{18}$.

\subsubsection{Practical consequences}

298 Increasing the sliding speed between the tablet and the die wall increases friction.

299 This has of course practical consequences in terms of tableting. First, it might explain 300 part of the strain rate sensitivity observed during compaction. Moreover, during 301 compaction, the sliding speed between the powder and die is not constant, both 302 during the compression and when considering the different parts of the tablets.

303 During compression, as the punches have to move backward, punch speed is 304 decreasing when approaching the compression top. This means that the friction 305 coefficient is of course not a constant during the compression event. More precisely, 306 when approaching the compaction top, as the speed decreases, friction also 307 decreases. This result might explain why most of the time, the friction coefficient 308 measured in the literature are decreasing during the compaction event ${ }^{19,20}$.

309 Moreover, for example in a symmetrical compaction, the center of the tablet is fixed, 310 whereas the parts in contact with the punches are moving at the punch velocity. 311 Between these two parts of the tablet, there is a speed gradient. This means that 
312 considering a constant coefficient of friction for the interaction between the die and

313 the tablet is clearly an oversimplification of the reality.

\section{Conclusion}

315 In this work, we demonstrated that the kinematic friction coefficient between the

316 tablet and the die during compaction is dependent on the sliding speed of the powder

317 on the surface. Thanks to the methodology developed, we were able to prove that

318 this effect is obtained independently from the compaction phase used to make the

319 tablet, i.e. it represents an intrinsic property of the friction between a tablet and a die 320 lubricated using magnesium stearate.

321 As a consequence, friction between the tablet and the die is dependent on the 322 compaction kinematics used and also changes during a compaction experiment 323 because the sliding speed between the tablet/powder and the die is not constant 324 during the compaction. It is also not the same at all the points of the tablets. This 325 effect may have consequences in the stress evolution during compaction, especially 326 in terms of strain rate sensitivity.

\section{Acknowledgement}

328 The authors acknowledge the support of the French Agence Nationale de la 329 Recherche (ANR), under grant ANR-17-CE08-0015 (project CliCoPha).

\section{Legend to figures}

331 Figure 1: Schematic representation of the protocol for friction measurement. UP: 332 upper punch, LP: lower punch 
333 Figure 2: Force on the upper punch during the pushing of the tablet in the die for

334 three different punch speeds.

335 Figure 3: Evolution of the force needed to move the tablet in the die $\left(F_{p}\right)$ as a 336 function of the upper punch speed for the four products. First compression (a) 337 150MPa and (b) 250MPa.

338 Figure 4: evolution of the die wall pressure as a function of the force on the upper 339 punch. First compression (a) 150MPa and (b) 250MPa.

340 Figure 5: Evolution of the friction coefficient as a function of the punch speed. First 341 compression (a) $150 \mathrm{MPa}$ and (b) $250 \mathrm{MPa}$.

342 Figure 6: Influence of the pressure used to make the tablet on the evolution of the 343 friction coefficient with the speed. (a) SDMan, (b)CaSul, (c) GLac, (d) ACP.

344 Figure 7: SEM photographs of the band of tablet obtained under 150MPa: (a) GLac,

345 (b) SDMan (c) CaSul and(d) ACP.

346 Figure 8: Photograph of the band of an ACP tablet obtained under 150MPa.

347 References

348 1. Armstrong NA. Time-dependent factors involved in powder compression and tablet 349 manufacture. Int J Pharm. 1989;49(1):1-13.

350 2. Çelik M, Aulton ME. The Viscoelastic Deformation of Some Tableting Materials as 351 Assessed by Indentation Rheology. Drug Dev Ind Pharm. 1996;22(1):67-75.

352 3. Katz JM, Buckner IS. Characterization of strain rate sensitivity in pharmaceutical 353 materials using indentation creep analysis. Int J Pharm. 2013;442(1):13-19.

4. Malamataris S, Rees JE. Viscoelastic properties of some pharmaceutical powders compared using creep compliance, extended Heckel analysis and tablet strength measurements. Int J Pharm. 1993;92(1-3):123-135.

357 5. Morehead WT. Viscoelastic Behavior of Pharmaceutical Materials During Compaction. 358 Drug Dev Ind Pharm. 1992;18(6-7):659-675. 
6. Radebaugh GW, Babu SR, Bondi JN. Characterization of the viscoelastic properties of compacted pharmaceutical powders by a novel nondestructive technique. Int J Pharm. 1989;57(2):95-105.

7. Rees JE, Rue PJ. Time-Dependent Deformation of Some Direct Compression Excipients. J Pharm Pharmacol. 1978;30(10):601-607.

8. Rippie EG, Danielson DW. Viscoelastic stress/strain behavior of pharmaceutical tablets: Analysis during unloading and postcompression periods. J Pharm Sci. 1981;70(5):476482.

9. Garr JSM, Rubinstein MH. An investigation into the capping of paracetamol at increasing speeds of compression. Int J Pharm. 1991;72(2):117-122.

10. Casahoursat L, Lemagnen G, Larrouture D. The Use of Stress Relaxation Trials to Characterize Tablet Capping. Drug Dev Ind Pharm. 1988;14(15-17):2179-2199.

11. Sun CC. Dependence of ejection force on tableting speed-A compaction simulation study. Powder Technol. 2015;279:123-126.

12. Campen S, Green J, Lamb G, Atkinson D, Spikes H. On the Increase in Boundary

13. Persson B. Sliding Friction - Physical Principles and Applications. Springer-Verlag Friction with Sliding Speed. Tribol Lett. 2012;48(2):237-248.

14. Moody G, Rubinstein MH, FitzSimmons RA. Tablet lubricants I. Theory and modes of

16. Briscoe BJ, Tabor D. Shear Properties of Thin Polymeric Films. J Adhes. 1978;9(2):145155.

17. Briscoe B. J., Evans D. C. B., Tabor David. The shear properties of Langmuir-Blodgett layers. Proc R Soc Lond Math Phys Sci. 1982;380(1779):389-407.

18. Zakowiecki D, Lachman M. Beyond just a filler - application of calcium phosphates in direct compression formulations. Express Pharma. 2017;December 1-15:90-92.

19. Sinka I.C., Cunningham J.C., Zavaliangos A. The effect of wall friction in the compaction of pharmaceutical tablets with curved faces: a validation study of the Drucker-Prager Cap model. Powder Technol. 2003;133(1):33-43.

20. Diarra H, Mazel V, Boillon A, et al. Finite Element Method (FEM) modeling of the powder compaction of cosmetic products: Comparison between simulated and experimental results. Powder Technol. 2012;224:233-240. 
(1)

(2)

(3)
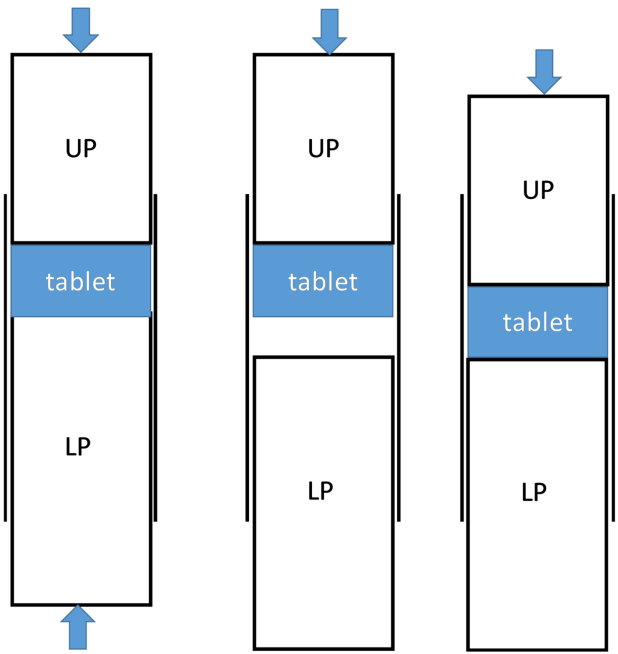


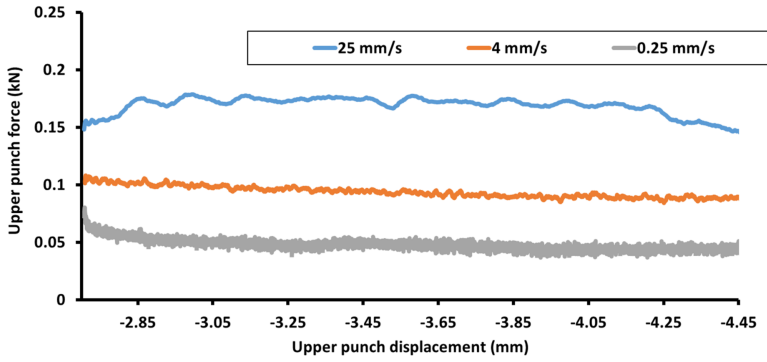




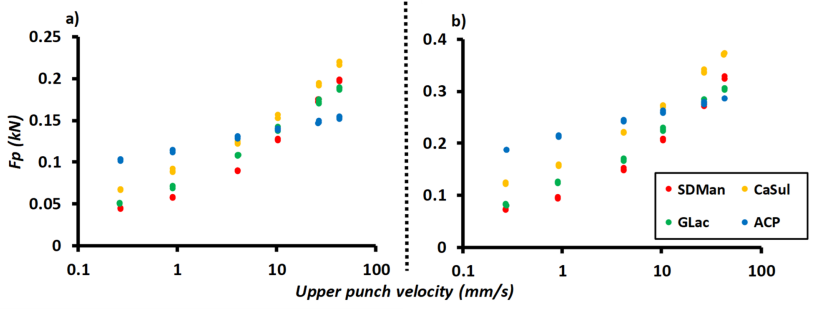




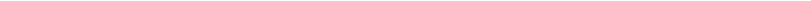




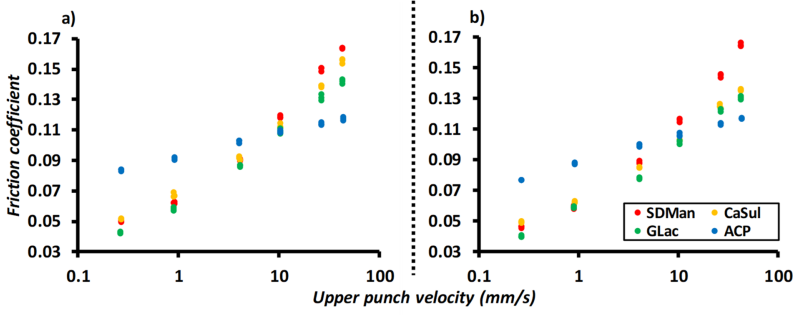



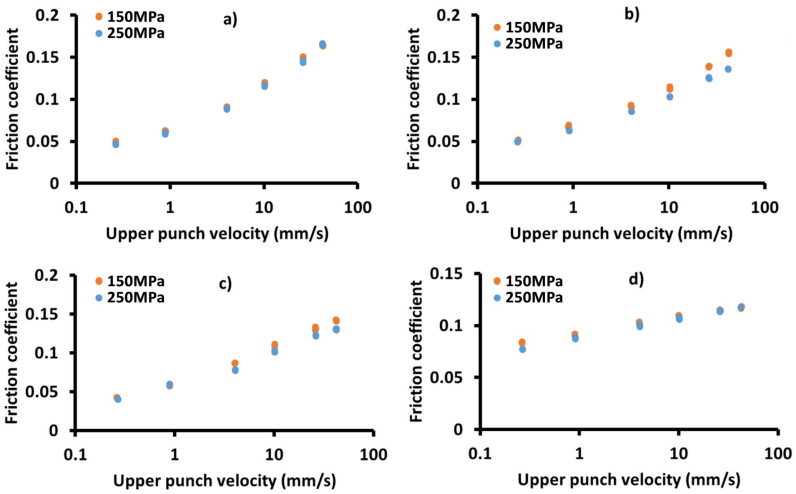


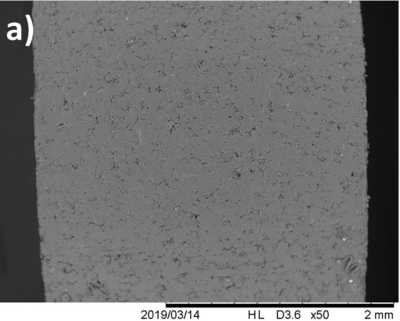

\section{b)}

(2019/03/1,

HL D3.6 $\times 50$

2019/03/14

HL D3.6 x50

$2 \mathrm{~mm}$

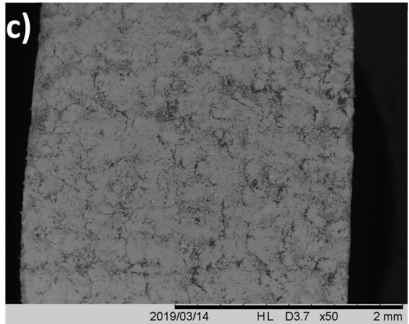

d

d)

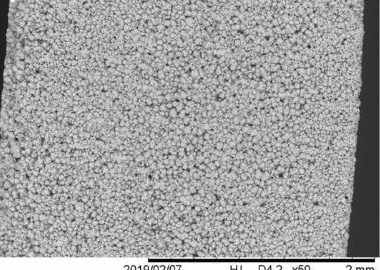

$2019 / 02 / 07$

HL D4.2 ×50

$2 \mathrm{~mm}$ 


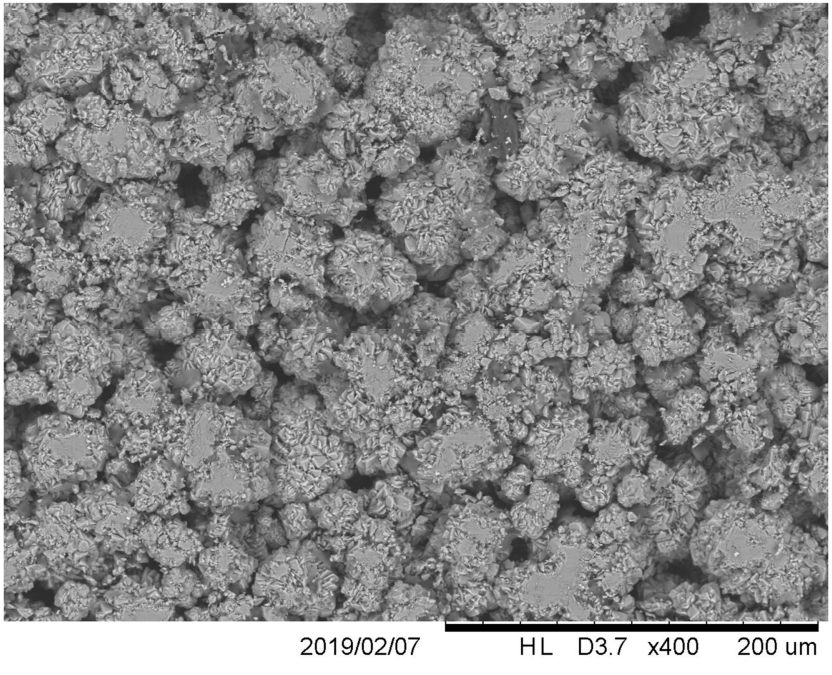

